\title{
The generation of Continuous-Variable Entanglement Frequency Comb
}

\section{SUBJECT AREAS:}

QUANTUM

INFORMATION

QUANTUM OPTICS

Received

16 October 2014

Accepted

17 December 2014

Published

20 January 2015

Correspondence and requests for materials should be addressed to

Y.B.Y. (ybyu@163.

com)
Youbin Yu' , Xiaomin Cheng' ${ }^{1}$, Huaijun Wang ', Zhongtao Shi' , Junwei Zhao', Fengmin Ji', Zhi Yin ${ }^{2}$ \& Yajuan Wang'

${ }^{1}$ School of Materials, Ningbo University of Technology, Ningbo 315211 , China, ${ }^{2}$ School of Sciences, Ningbo University of Technology, Ningbo 31521 1, China.

Continuous-variable (CV) entanglement frequency comb can be produced by enhanced Raman scattering in an above-threshold optical oscillator cavity in which a hexagonally-poled $\mathrm{LiTaO}_{3}$ crystal resides as a Raman gain medium. The Stokes and anti-Stokes Raman signals are enhanced by a coupled quasi-phase-matching optical parametric process and the entanglement natures among these Raman signals and pump are demonstrated by applying a sufficient inseparability criterion for CV entanglement. Such entanglement frequency comb source with different frequencies and continuously tunable frequency interval may be very significant for the applications in quantum communication and networks.

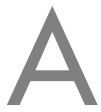

valuable feature of quantum optical implementations based on continuous-variable (CV) entanglement, related to their high efficiency, is their unconditionalness ${ }^{1}$. Multipartite $\mathrm{CV}$ entangled beams, produced generally by combining squeezed beams on linear beam splitters, are degenerate ${ }^{2-5}$. However, multipartite $\mathrm{CV}$ entangled beams with different frequencies are necessary to connect different physical systems at the nodes of quantum networks ${ }^{6}$ since they can be separated easily in the systems. In contrast to the degenerate case, multicolor entanglement has to be generated directly by nonlinear optical processes. It was predicted that two-color $\mathrm{CV}$ entanglement can be produced by the nondegenerate optical parametric oscillator (NOPO) ${ }^{7}$, which has been demonstrated experimentally below ${ }^{8}$, as well as above ${ }^{9}$, the oscillation threshold. Recently, theory predicts that three-color CV entanglement can be generated by operating an OPO above its threshold ${ }^{10}$, which has been verified in a recent experiment ${ }^{11}$. The three-color $\mathrm{CV}$ entanglement beams can also be produced by quasiphase-matching $(\mathrm{QPM})^{12,13}$ third-harmonic generation ${ }^{14}$ or forth-harmonic generation ${ }^{15}$, respectively. The bright quadricolor $\mathrm{CV}$ entanglement can be generated by four-wave-mixing process ${ }^{16}$.

Optical frequency comb (FC) can be generated from multimode OPO, which plays an important role in the application of one-way quantum computation ${ }^{17}$. More recently, a scheme was proposed to produce large $\mathrm{CV}$ cluster state using one single-mode vacuum squeezer and a quantum nondemolition gate ${ }^{18}$, and another scheme was presented to create multioctave FC by stimulated Raman scattering (RS) in a hydrogen-filled hollow-core photonic crystal fiber ${ }^{19}$. CV cluster states can also produced in the time domain ${ }^{20}$. The multipartite entanglement of 60 modes of a quantum optical FC was experimental generated in a bimodally pumped OPO ${ }^{21}$. The quantum frequency comb is easily addressable, robust with respect to decoherence and scalable, which renders it a unique tool for quantum information ${ }^{22}$.

In the present paper, we propose a new scheme to directly produce multicolor CV entanglement FC by enhanced RS in an above-threshold optical oscillator cavity, in which a two-dimensional hexagonally-poled $\mathrm{LiTaO}_{3}$ (2D-HPLT) crystal serves as a Raman gain medium. This idea is based on the previous experimental result of Ref. 23 and the elicitation derived from the beautiful experiment of Ref. 11. In this case, we verify, for the first time, that all the Stokes and anti-Stokes Raman frequency components in this comb, including the pump, are mutually entangled by applying the sufficient inseparability criterion for CV entanglement ${ }^{24}$. This scheme of generating multicolor CV entanglement FC comes directly from a single optical superlattice inside the optical cavity, which has been achieved experimentally without the optical cavity ${ }^{23}$, and exhibited a symmetrical FC of phonon-polariton (P-P) RS enhanced by a coupled QPM optical parametric amplification processes.

\section{Enhanced RS.}

We consider a 2D-HPLT crystal as a Raman gain medium located inside an one-sided optical oscillator cavity, as illustrated in Fig.1(a). Initially, a pump with the frequency of $\omega_{0}$ and a signal with the frequency of $\omega_{1}$ are incident onto the crystal ${ }^{23}$. Then, a beam with the frequency of $\omega_{-1}$ is generated by the difference frequency generation 
between the pump and signal beams, and the phase mismatching is compensated by the reciprocal $\mathrm{G}$ via a process of QPM as given by

$$
\mathbf{k}_{0}=\mathbf{k}_{1}+\mathbf{k}_{-1}+\mathbf{G} \text {. }
$$

The intense P-P field supplies the inelastic source and generates the anti-Stokes beams

$$
\omega_{q}=\omega_{1}+(q-1) \omega_{p}
$$

and Stokes beams

$$
\omega_{-q}=\omega_{-1}-(q-1) \omega_{p},
$$

where $q(=1,2,3 \cdots$,$) stands for the order of the RS.$

Figure 1(b) gives the sketched momentum geometry for the QPMOPA. The high-order RS beams can be significantly amplified by the pump through the process of coupled QPM-OPA, which is related to the second-order nonlinear interaction, and induces the rapid growth of the RS signals ${ }^{25}$. In the previous experiment, the eighthorder RS beam was observed and the relative intensities of the RS beams approach to $14 \%-70 \%$. For example, Fig. 1(c) shows the Raman spectrum which was obtained in the previous experiment ${ }^{23}$. One can see that the Stokes and anti-Stokes frequency components distribute symmetrically and form a comb-like structure with equal frequency interval.

\section{Output fields}

The interaction Hamiltonian for the coupled nonlinear processes can be written as $^{26}$

$$
\hat{H}_{I}=i \hbar \sum_{q}\left(\hat{a}_{0} \hat{a}_{q}^{\dagger} \hat{a}_{-q}^{\dagger}+\kappa_{q} \hat{a}_{q} \hat{a}_{q+1}^{\dagger}+\kappa_{-q} \hat{a}_{-q} \hat{a}_{-q-1}^{\dagger}\right)+\text { h.c. },
$$

where $\hat{a}_{0}, \hat{a}_{q}$ and $\hat{a}_{-q}(q=1,2,3, \cdots)$ are annihilation operators for the cavity modes, respectively. $\kappa_{0}, \kappa_{q}$ and $\kappa_{-q}$ are the effective coupling constants, which are proportional to the nonlinear susceptibility and structure parameters, and taken to be real without loss of generality $^{27-29}$.

The equations of motion for the cavity modes can be obtained by solving the Fokker-Planck equation in the positive- $P$ representa- tion $^{30-33}$. In this case, for example, we only consider the first- and second-order RS beams in the cavity. Then, one can obtain the equations of motion of the five cavity modes as ${ }^{30,31}$

$$
\begin{aligned}
& \partial \alpha_{0} / \partial t=\varepsilon_{0}-\gamma_{0} \alpha_{0}-\kappa_{0} \alpha_{1} \alpha_{-1}-\kappa_{0} \alpha_{2} \alpha_{-2}, \\
& \partial \alpha_{1} / \partial t=\varepsilon_{1}+\kappa_{0} \alpha_{0} \alpha_{-1}^{\dagger}-\gamma_{1} \alpha_{1}-\kappa_{1} \alpha_{2}+\sqrt{\kappa_{0} \alpha_{0}} \eta_{1}(t), \\
& \partial \alpha_{-1} / \partial t=\kappa_{0} \alpha_{0} \alpha_{1}^{\dagger}-\gamma_{-1} \alpha_{-1}-\kappa_{-1} \alpha_{-2}+\sqrt{\kappa_{0} \alpha_{0}} \eta_{2}(t), \\
& \partial \alpha_{2} \partial t=\kappa_{0} \alpha_{0} \alpha_{-2}^{\dagger}-\gamma_{2} \alpha_{2}+\kappa_{2} \alpha_{1}+\sqrt{\kappa_{0} \alpha_{0}} \eta_{3}(t), \\
& \partial \alpha_{-2} / \partial t=\kappa_{0} \alpha_{0} \alpha_{2}^{\dagger}-\gamma_{-2} \alpha_{-2}+\kappa_{-2} \alpha_{-1}+\sqrt{\kappa_{0} \alpha_{0}} \eta_{4}(t),
\end{aligned}
$$

where $\alpha_{i}$ and $\alpha_{i}^{\dagger}$ correspond to the mode operators $a_{i}$ and $a_{i}^{\dagger}$ in the positive-P representation, respectively, $\gamma_{i}(\mathrm{i}=0, \pm 1, \pm 2)$ stand for the damping rates for the corresponding cavity modes, $\varepsilon_{0}$ and $\varepsilon_{1}$ are the classical pump laser amplitudes, and $\eta_{i}$ are complex Gaussian noise terms with $\left\langle\eta_{i}(t)\right\rangle=\left\langle\eta_{i}^{\dagger}(t)\right\rangle=0$ and $\left\langle\eta_{i}(t) \eta_{j}\left(t^{\prime}\right)\right\rangle=$ $\left\langle\eta_{i}^{\dagger}(t) \eta_{j}^{\dagger}\left(t^{\prime}\right)\right\rangle=\delta_{i j} \delta\left(t-t^{\prime}\right)$. In order to simplify the calculation we assume $\gamma_{1}=\gamma_{-1}=\gamma_{2}=\gamma_{-2}=\gamma$ and $\kappa_{2}=\kappa_{-2}=\kappa_{-1}=\kappa_{1}$ in the above equations. Also, we consider the pump and injected signal as real fields, i. e. $\varepsilon_{0}=\varepsilon_{0}^{*}=E_{0}$ and $\varepsilon_{1}=\varepsilon_{1}^{*}=E_{1}$.

The steady-state solutions can be obtained by letting $\partial \alpha_{i} / \partial t=0(i$ $=0, \pm 1, \pm 2$ ) and ignoring the noise terms in Eq. (5). The steadystate value $A_{0}$ of the pump satisfies the following equation

$$
\Delta_{0}\left(\kappa_{0}^{2} A_{0}^{2} \Delta_{1}+\Delta_{2}^{2}\right)+E_{1}^{2} \kappa_{0}^{2} \gamma A_{0}=0,
$$

where $\Delta_{0}=\gamma_{0} A_{0}-E_{0}, \Delta_{1}=\kappa_{0}^{2} A_{0}^{2}+2\left(\kappa_{1}^{2}-\gamma^{2}\right)$, and $\Delta_{2}=\kappa_{1}^{2}+\gamma^{2}$. The other steady-state values are related to $A_{0}$ as $A_{1}=$ $\frac{1}{E_{1} \Delta_{2}}\left[E_{1}^{2} \gamma+\Delta_{0}\left(\Delta_{1}+\Delta_{2}\right) A_{0}\right], \quad A_{2}=\frac{\kappa_{1}}{E_{1} \gamma \Delta_{2}}\left[E_{1}^{2} \gamma+\Delta_{0}\left(\Delta_{1}-\Delta_{2}\right) A_{0}\right]$, $A_{-1}=\frac{\Delta_{0}}{2 E_{1} \kappa_{0} \gamma}\left(\Delta_{1}+\kappa_{0}^{2} A_{0}^{2}\right)$, and $A_{-2}=-\frac{2 \kappa_{1}}{E_{1} \kappa_{0}} \Delta_{0}$, where $A_{i}(i=$ $\pm 1, \pm 2)$ are the steady-state values of the cavity modes. Because the Eq. (6) is a fifth-order equation, it is well known, there is no analytical solutions for it. So one can not obtained the threshold of the system although one can solve the Eq. (6) by numerical calculation. In order to discuss expediently, we use $\varepsilon_{t h}=\gamma_{0} \gamma / \kappa_{0}$, which is the well-known threshold for the OPO, as a terminology for the threshold. Generally, the injected signal $\omega_{1}$ can increase the conversion (a)

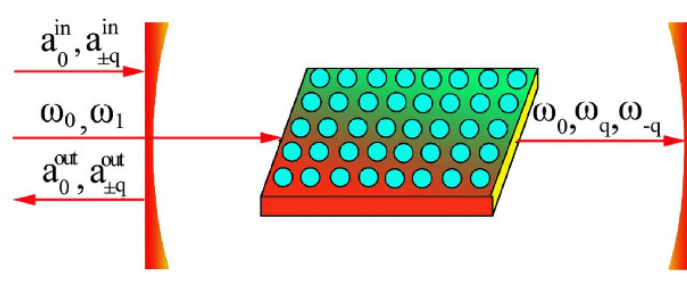

(c)

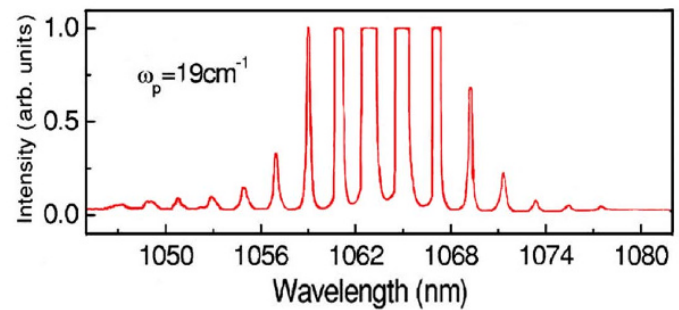

(b)

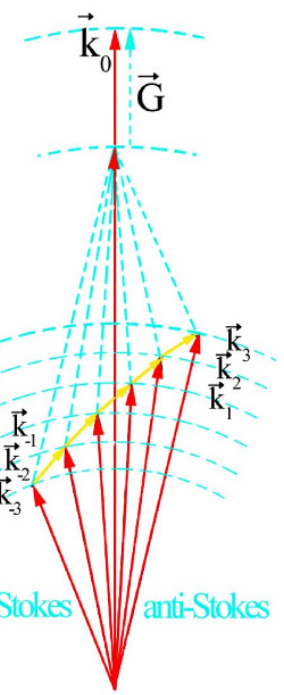

Figure $\mathbf{1} \mid$ (a) Sketch of the one-sided cavity. (b) The sketched momentum geometry of a coupled quasi-phase-matching process. (c) The Raman spectra corresponding P-P frequency $\omega_{p}=19 \mathrm{~cm}^{-123}$. 
efficiency and stability, and change the characteristics of the threshold.

In the following, one can decompose the system variables into their steady-state values and small fluctuations around the steadystate values as $\alpha_{i}=A_{i}+\delta \alpha_{i}$ and use this linearized analysis as a method to rewrite the evolution equations in Eq. (5) as ${ }^{30,31}$

$$
d \delta \tilde{\alpha}=-\mathbf{M} \delta \tilde{\alpha} d t+\mathbf{B} d W
$$

where $\delta \tilde{\alpha}=\left[\delta \alpha_{0}, \delta \alpha_{0}^{\dagger}, \delta \alpha_{1}, \delta \alpha_{1}^{\dagger}, \delta \alpha_{-1}, \delta \alpha_{-1}^{\dagger}, \delta \alpha_{2}, \delta \alpha_{2}^{\dagger}, \delta \alpha_{-2}, \delta \alpha_{-2}^{\dagger}\right]^{\mathrm{T}} ; \mathbf{M}$ is the drift matrix with the steady-state values; $\mathbf{B}$ contains the steady-state coefficients of the noise terms of Eq. (5), and $d W$ is a vector of Wiener increment ${ }^{30,31}$. The condition for the validity of the linearization process is that the eigenvalues of the drift matrix $M$ have no negative real parts ${ }^{30,31}$. The analytical expressions since the eigenvalues of $\mathbf{M}$ can't be obtained here for the fifth equation has no analytical solutions. However, we find the eigenvalues are related to $\kappa_{1}$ via numerical calculation. When $\kappa_{1} \geq \kappa_{1 \text { th }}$, where $\kappa_{1 t h}=2 E_{1} \kappa_{0} / \sqrt{\gamma_{0} \gamma}$, the negative real parts only present in the range of $\varepsilon_{0} \geq \varepsilon_{t h}$. With the decrease of $\kappa_{1}$, when $\kappa_{1}<\kappa_{1 t h}$, the positive real parts become present in the range of $\varepsilon_{0} \geq \varepsilon_{\mathrm{th}}$. In Fig. 2 We plot the real parts of the eigenvalues versus the pump power parameter $\varepsilon_{0} / \varepsilon_{\text {th }}$ with $\gamma_{0}=0.03, \gamma=0.01, \kappa_{0}=0.05$, and $E_{1}=2 \gamma \sqrt{2 \gamma_{0} \gamma} / \kappa_{0}$ for $\kappa_{1}=$ $10 \kappa_{1 t h}, \kappa_{1 t h}, 0.1 \kappa_{1 t h}$, and $0.01 \kappa_{1 t h}$, respectively. In present study, we only focus on the OPO operating above the threshold. From Fig. 2 one can see that there are positive real parts appear in the range of above the threshold only when $\kappa_{1}<\kappa_{1 \text { th }}$ is satisfied. In addition, there are negative real parts appear at about $\varepsilon_{0} / \varepsilon_{t h}=6.8$ in Fig. 2(c). With the further decrease of $\kappa_{1}$, when $\kappa_{1}=0.01 \kappa_{1 t h}$, one can see from Fig. 2(d) that the real parts of the eigenvalues are all above zero in the range of $\varepsilon_{0} \leq 10 \varepsilon_{t h}$. Above analysis indicates that the linearization analysis is valid in the case depicted in Fig. 2(c) and (d).

Then, from Eq. (7) one can obtain the intracavity spectral matrix in the frequency domain as ${ }^{30,31}$

$$
\mathbf{S}(\omega)=(\mathbf{M}+i \omega \mathbf{I})^{-1} \mathbf{B B}^{\mathrm{T}}\left(\mathbf{M}^{\mathrm{T}}-i \omega \mathbf{I}\right)^{-1},
$$

where $\mathbf{I}$ is the identity matrix and $\omega$ is the analysis frequency. By applying the well-known input-output relations ${ }^{34}$ one can obtain the output fields.
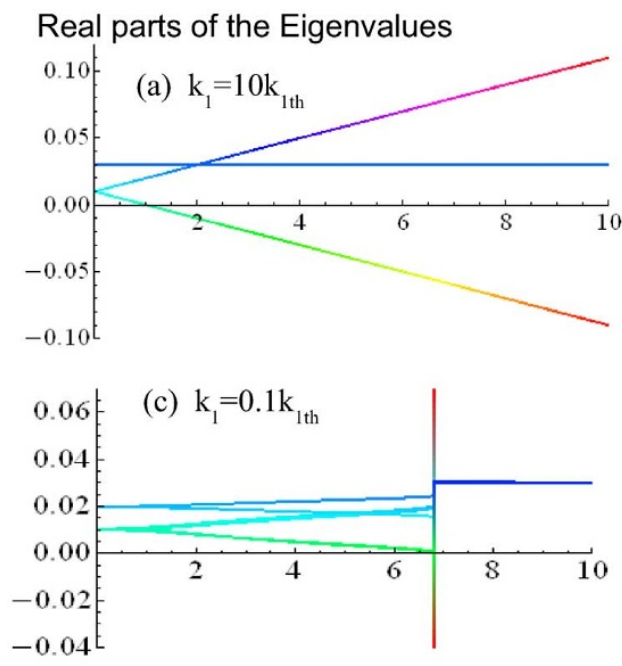

\section{CV entanglements in the FC}

When $q=1$, there are three modes in the cavity, the experiment setup is exactly similar to an above-threshold OPO with an injected signal which has been demonstrated that the three modes, i. e. pump, signal, and idler, are CV entangled with each other ${ }^{35}$. So when $q=1$, one only considers the first order RS, the three modes, i. e. $\hat{a}_{0}, \hat{a}_{1}$, and $\hat{a}_{-1}$, are CV entangled with each other when the cavity operates above the oscillation threshold. When $q=2$, there are five modes in the cavity including two pairs of RS beams and the pump. From the multipartite CV entanglement criteria ${ }^{24}$, four of the simultaneous satisfactions in the following inequalities

$$
\begin{aligned}
& V_{0,1}=\Delta^{2}\left(\hat{X}_{0}+\hat{X}_{1}\right)+\Delta^{2}\left(\hat{Y}_{0}-\hat{Y}_{1}+g_{-1} \hat{Y}_{-1}+g_{2} \hat{Y}_{2}+g_{-2} \hat{Y}_{-2}\right)<1, \\
& V_{1,-1}=\Delta^{2}\left(\hat{X}_{1}-\hat{X}_{-1}\right)+\Delta^{2}\left(g_{0} \hat{Y}_{0}+\hat{Y}_{1}+\hat{Y}_{-1}+g_{2} \hat{Y}_{2}+g_{-2} \hat{Y}_{-2}\right)<1, \\
& V_{-1,2}=\Delta^{2}\left(\hat{X}_{-1}-\hat{X}_{2}\right)+\Delta^{2}\left(g_{0} \hat{Y}_{0}+g_{1} \hat{Y}_{1}+\hat{Y}_{-1}+\hat{Y}_{2}+g_{-2} \hat{Y}_{-2}\right)<1,(9) \\
& V_{2,-2}=\Delta^{2}\left(\hat{X}_{2}-\hat{X}_{-2}\right)+\Delta^{2}\left(g_{0} \hat{Y}_{0}+g_{1} \hat{Y}_{1}+g_{-1} \hat{Y}_{-1}+\hat{Y}_{2}+\hat{Y}_{-2}\right)<1, \\
& V_{-2,0}=\Delta^{2}\left(\hat{X}_{-2}+\hat{X}_{0}\right)+\Delta^{2}\left(\hat{Y}_{-2}-\hat{Y}_{0}+g_{1} \hat{Y}_{1}+g_{-1} \hat{Y}_{-1}+g_{2} \hat{Y}_{2}\right)<1,
\end{aligned}
$$

should be sufficient to verify the five-mode CV entanglement. In addition, the first two of the simultaneous satisfactions in above inequalities should be sufficient to demonstrate $a_{0}, a_{1}$, and $a_{-1}$ three-mode $\mathrm{CV}$ entanglement when five-mode interaction exists. $\hat{X}_{i}=\left(\alpha_{i}^{\text {out }}+\alpha_{i}^{\text {out } \dagger}\right) / 2(i=0, \pm 1, \pm 2)$ represent the amplitude quadratures of the fields; $\hat{Y}_{i}=\left(\alpha_{i}^{\text {out }}-\alpha_{i}^{\text {out } \dagger}\right) / 2 i$ stand for their phase quadratures; gi are the adjustable parameters chosen to minimize the left of the inequalities.

In Fig. 3(a) and (b) we show that the minima of the five inequalities versus the analysis frequency $\omega$ with $\gamma_{0}=0.03, \gamma=0.01, \kappa_{0}=0.05, \varepsilon_{0}$ $=E_{0}=2 \varepsilon_{t h}$, and $E_{1}=2 \gamma \sqrt{2 \gamma_{0} \gamma} / \kappa_{0}$ for different values of $\kappa_{1}: \kappa_{1}=$ $0.1 \kappa_{1 t h}$ and $\kappa_{1}=0.01 \kappa_{1 t h}$, respectively. One can see in Fig. 3(a) and (b) that the minima of the five inequalities are all below 1 in a wide range of analysis frequency and Eq. (9) is satisfied, which indicates that the two pairs of RS beams and the pump are CV entangled with each other. In addition, the results in Fig. 3(a) and (b) indicate that the entanglement nature of the same-order RS beams is stronger than that between the pump and RS beams as well as among differentorder RS beams. In fact, when the optical cavity operates below the threshold value, we don't need to consider the quantum nature of the

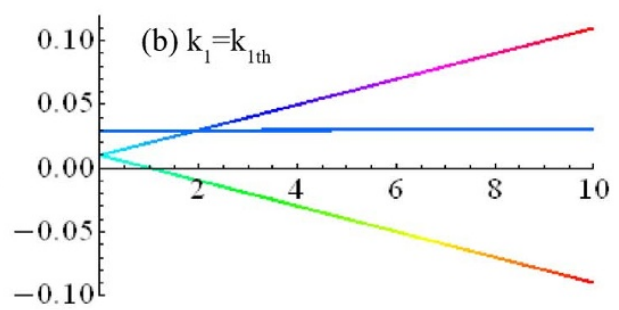

(d) $\mathrm{k}_{1}=0.01 \mathrm{k}_{1 \mathrm{t}}$

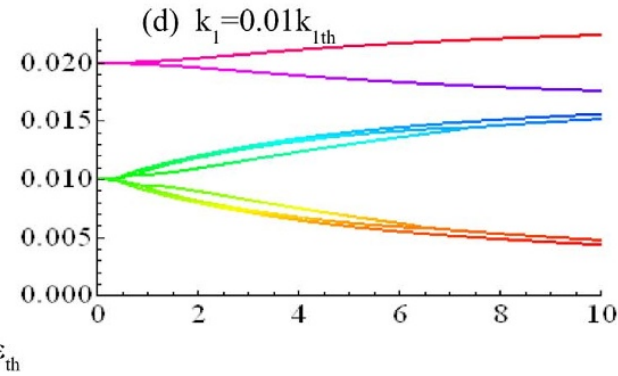

Figure $2 \mid$ The real parts of the eigenvalues versus the pump power parameter $\varepsilon_{0} / \varepsilon_{t h}$ for different $\kappa_{1}:(a) \kappa_{1}=10 \kappa_{1 t h} ;(b) \kappa_{1}=\kappa_{1 t h} ;(c) \kappa_{1}=0.1 \kappa_{1 t h} ;(d) \kappa_{1}$ $=0.01 \kappa_{1 t h}$. 

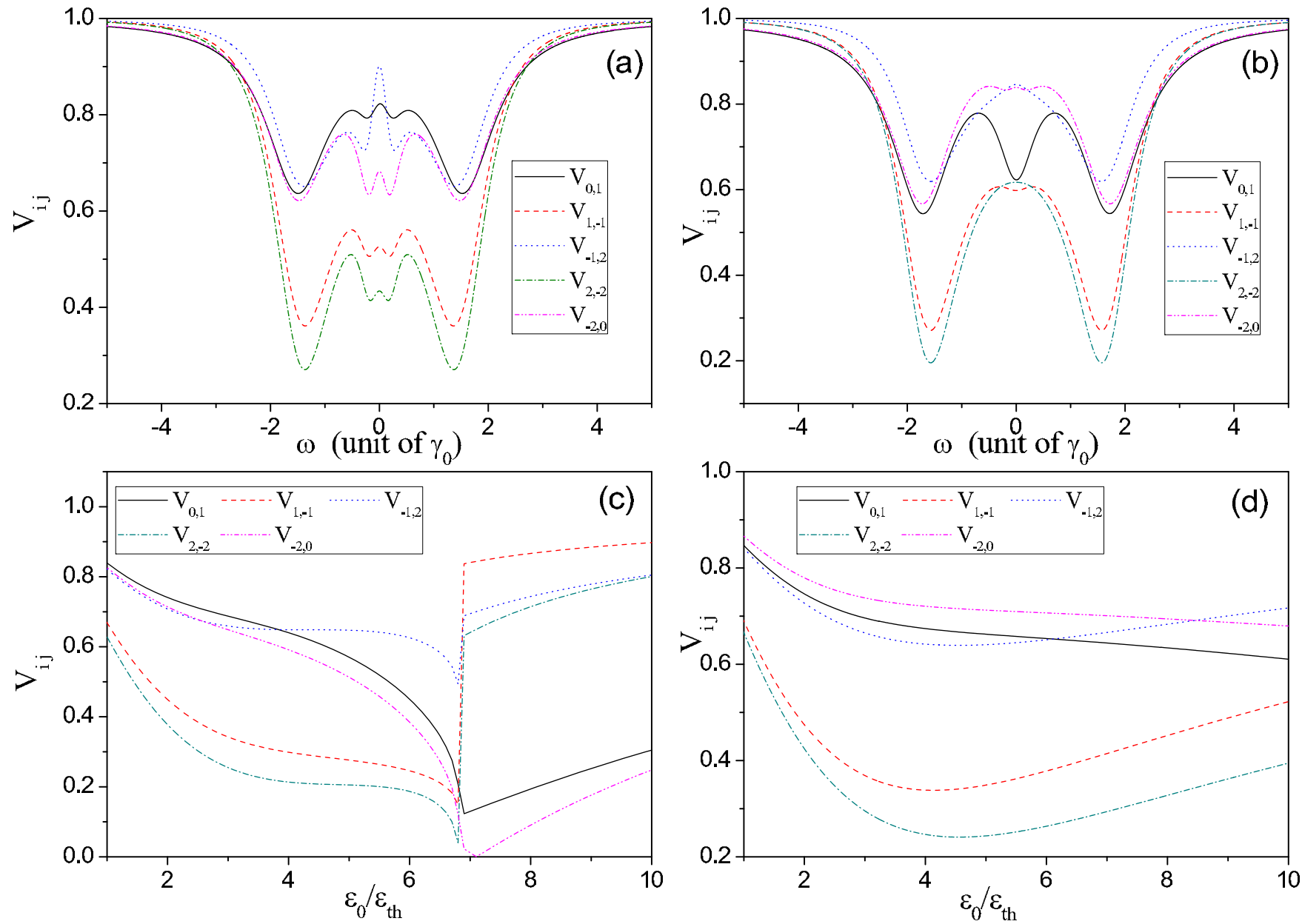

Figure $3 \mid$ The minima of the inequalities versus the analysis frequency $\omega$ for different values of $\kappa_{1}:(a) \kappa_{1}=0.1 \kappa_{1 t h} ;(b) \kappa_{1}=0.01 \kappa_{1 t h}$. The minima of five inequalities versus the pump power parameter $\varepsilon_{0} / \varepsilon_{t h}$ for different values of $\kappa_{1}$ : (c) $\kappa_{1}=0.1 \kappa_{1 t h}$; (d) $\kappa_{1}=0.01 \kappa_{1 t h}$.

pump beam since it is undepleted, and only the same-order RS beams are CV entangled ${ }^{36}$. When $\varepsilon>\varepsilon_{t h}$, the damping of the pump beam can't be ignored, and the quantum nature of the pump should be included. The same-order RS beams are both CV entangled with the pump for the energy conservation $\omega_{0}=\omega_{q}+\omega_{-q}(q=1,2,3 \cdots)$ and they are amplified through coupled QPM optical parametric processes. The adjacent-order scattering beams should not be entangled because RS is a kind of inelastic scattering process ${ }^{16}$. However, they are entangled as shown in Fig. 3(a) and (b), the reason for which is that they are both entangled with the pump beam, i. e. the adjacentorder RS beams are CV entangled via the entanglement transfer through the pump beam.

When $\kappa_{1}=0$, only the first RS exists, the three fields of $a_{0}, a_{1}$, and $a_{-1}$ are CV entangled with each other since it is similar to an abovethreshold OPO with an injected signal which we have investigated demonstrated that the three fields are CV entangled ${ }^{35}$. From Figs. $3(\mathrm{a})$ and (b) one also can see that the first two inequalities are simultaneous satisfied which indicates that the three fields of $a_{0}, a_{1}$, and $a_{-1}$ are $\mathrm{CV}$ entangled with each other when the five-mode interaction exists. The extended nonlinear interaction do not influence the quantum entanglement nature of the RS beams.

In addition, the degree of entanglement also depends on the intensity difference among the beams. Smaller the intensity difference is, stronger the quantum correlation will be. With the increase of the order of RS, the quantum correlation between the high-order Raman signals and the center of the comb will decrease. Figure 1c), for example, depicts that the Raman beams exhibit a comb-shaped structure with an equal frequency interval which was obtained in the previous experiment for the single-pass nonlinear process ${ }^{23}$. The relative intensities for the first- to eighth-order Raman signals are obtained to be from $14 \%$ to $70 \%$. However, if this setup is placed into an optical cavity, the relative intensity will be significantly enhanced. Then, the quantum correlations between the high-order Raman signals and the center of the comb will increase. In fact, the same order Raman signals are both entangled with the pump beam by the optical parametric amplification process according to Refs. 10,11, 35, and the adjacent-order Raman signals are also entangled via the entanglement transfer through the pump beam. Therefore, even more optical sidebands are added in the Raman interactions, all the RS signals and the pump are entangled with each other, although the larger intensity difference can decrease the quantum correlation between the high-order RS signals and the pump. Based on above analysis, the demonstrations of three-mode and five-mode entanglement should be enough to verify all the RS beams are entangled with each other. Ultimately, in this scheme a single optical superlattice can directly generate bright multicolor $\mathrm{CV}$ entangled $\mathrm{FC}$, which involves multipartite $\mathrm{CV}$ entangled beams with different frequencies, having a continuously tunable and equal frequency interval.

Figure 3(c) and (d) depict the minima of the five inequalities versus the pump power parameter $\varepsilon_{0} / \varepsilon_{t h}$ with $\kappa_{1}=0.1 \kappa_{1 \text { th }}$ and $\kappa_{1}$ $=0.01 \kappa_{1 t h}$, respectively. One can see from Fig. $3(\mathrm{c})$ and $(\mathrm{d})$ that the minima of the five inequalities are all below 1 in the wide range of pump power parameter which demonstrates the RS beams and pump are CV entangled each other when the optical cavity operates above its threshold. Moreover, one can see that the minima of the five inequalities decrease initially, then increase with the increase of $\varepsilon_{0}$. Initially, with increasing pump above the threshold, RS beams are enhanced, however, the energy of the pump will be transferred to the 
RS beams. Then, the quantum nature of the pump emerges and the bright multicolor CV entanglement FC can be obtained. With the further increase of the pump, the gain begins to saturate, so the intensity of the pump becomes larger than that of the RS beams, and then its quantum nature will vanish. In addition, there is an abrupt transition at about $\varepsilon_{0} / \varepsilon_{t h}=6.8$ in Fig. 3(c) which is good agreement with the case in Fig. 2(c). The negative eigenvalues of the drift matrix lead to the abrupt transition at this position for the abrupt increase of the fluctuations.

\section{In summary}

We proposed a new scheme to directly produce bright multicolor $\mathrm{CV}$ entanglement FC by enhanced RS in an optical cavity with a $2 \mathrm{D}$ HPLT crystal as the Raman gain medium. We have theoretically verified that the Stokes, anti-Stokes RS beams, and the pump are $\mathrm{CV}$ entangled by applying sufficient inseparability criteria for multipartite $\mathrm{CV}$ entanglement. The adjacent-order RS beams are CV entangled via the entanglement transfer through the pump beam. Because the single-pass process has been experimentally realized, the multimode squeezed light has been produced for both spatial ${ }^{40,41}$ and frequency ${ }^{42}$ modes, respectively, and the frequency intervals of the RS beams in this scheme are equal and continuously tunable, so it is experimentally feasible to design an optical cavity, in which all the beams oscillate simultaneously.

This entangled FC source, generated from optical supperlattice and with different frequencies and continuously tunable frequency interval and high beam intensity, may be very useful for the applications in quantum communication and networks. For example, the entangled frequency components could be drastically altered using interspecies quantum teleportation protocols, which would enhance the integrability of disparate nodes in a quantum information network ${ }^{6,37}$. Similar to the case in Ref. 17, the entangled state generated in the current scheme also has the equal frequency spacing and forms an optical frequency comb. Certainly, not all types of multipartite states are useful for quantum computing 38,39 . The quantum frequency comb is easily addressable, robust with respect to decoherence and scalable, which renders it a unique tool for quantum information.

In the previous experiment ${ }^{23}$, the eighth-order RS beam was observed and the relative intensities of the RS beams approach to $14 \%-70 \%$, since the Stokes and anti-Stokes RS beams are significantly enhanced by coupled QPM optical parametric processes. One can obtain at least 17 modes (including the pump) entanglement frequency comb in present scheme. However, when one take this device into an optical cavity, the RS beams intensity will be further enhanced, more high-order RS beams will be observed and these beams are also entangled with each other by the optical parametric processes. Moreover, the frequency intervals of the RS beams in this scheme are equal and continuously tunable. Therefore, comparing to the previously reported quantum optical frequency comb, this scheme should generate better quantum correlations because the utilization of QPM technique can greatly enhance both the Raman signal and the entanglement.

1. Braunstein, S. L. \& van Loock, P. Quantum information with continuous variables. Rev. Mod. Phys. 77, 513 (2005).

2. van Loock, P. \& Braunstein, S. L. Multipartite Entanglement for Continuous Variables: A Quantum Teleportation Network. Phys. Rev. Lett. 84, 3482 (2000).

3. Jing, J. T. et al. Experimental Demonstration of Tripartite Entanglement and Controlled Dense Coding for Continuous Variables. Phys. Rev. Lett. 90, 167903 (2003).

4. Aoki, T. et al. Experimental Creation of a Fully Inseparable Tripartite Continuous-Variable State. Phys. Rev. Lett. 91, 080404 (2003).

5. Giedke, G., Kraus, B., Lewenstein, M. \& Cirac, J. I. Separability properties of threemode Gaussian states. Phys. Rev. A 64, 052303 (2001)

6. Kimble, H. J. The quantum internet. Nature 453, 1023-1030 (2008).

7. Reid, M. D. \& Drummond, P. D. Quantum Correlations of Phase in Nondegenerate Parametric Oscillation. Phys. Rev. Lett. 60, 2731-2733 (1988).
8. Ou, Z. Y., Pereira, S. F., Kimble, H. J. \& Peng, K. C. Realization of the EinsteinPodolsky-Rosen paradox for continuous variables. Phys. Rev. Lett. 68, 3663-3666 (1992).

9. Villar, A. S., Cruz, L. S., Cassemiro, K. N., Martinelli, M. \& Nussenzveig, P. Generation of Bright Two-Color Continuous Variable Entanglement. Phys. Rev. Lett. 95, 243603 (2005)

10. Villar, A. S., Martinelli, M., Fabre, C. \& Nussenzveig, P. Direct Production of Tripartite Pump-Signal-Idler Entanglement in the Above-Threshold Optical Parametric Oscillator. Phys. Rev. Lett. 97, 140504 (2006).

11. Coelho, A. S. et al. Three-Color Entanglement. Science 326, 823-826 (2009).

12. Armstrong, J., Bloembergen, N., Ducuing, J. \& Pershan, P. S. Interactions between Light Waves in a Nonlinear Dielectric. Phys. Rev. 127, 1918-1939 (1962).

13. Zhu, S. N., Zhu, Y. Y. \& Ming, N. B. QuasiCPhase-Matched Third-Harmonic Generation in a Quasi-Periodic Optical Superlattice. Science 278, 843-846 (1997).

14. Yu, Y. B., Wang, H. J., Xiao, M. \& Zhu, S. N. Directly produced three-color entanglement by quasi-phase-matched third-harmonic generation. Optics Express 375, 2278 (2011).

15. Yu, Y. B. \& Wang, H. J. Bright three-color continuous-variable entanglement generated by a cascaded sum-frequency process in an optical cavity. J. Opt. Soc. Am. B 28, 1899 (2011).

16. Yu, Y. B., Sheng, J. T. \& Xiao, M. Generation of bright quadricolor continuousvariable entanglement by four-wave-mixing process. Phys. Rev. A 83, 012321 (2011).

17. Menicucci, N. C., Flammia, S. T. \& Pfister, O. One-Way Quantum Computing in the Optical Frequency Comb. Phys. Rev. Lett. 101, 130501 (2008).

18. Menicucci, N. C., Ma, X. \& Ralph, T. C. Arbitrarily Large Continuous-Variable Cluster States from a Single Quantum Nondemolition Gate. Phys. Rev. Lett. 104, 250503 (2010).

19. Wang, Y. Y., Wu, C. B., Couny, F., Raymer, M. G. \& Benabid, F. QuantumFluctuation-Initiated Coherence in Multioctave Raman Optical Frequency Combs. Phys. Rev. Lett. 105, 123603 (2010).

20. Yokoyama, S. et al. Ultra-large-scale continuous-variable cluster states multiplexed in the time domain. Nature Photon. 7, 982 (2013).

21. Chen, M., Menicucci, N. C. \& Pfister, O. Experimental Realization of Multipartite Entanglement of 60 Modes of a Quantum Optical Frequency Comb. Phys. Rev. Lett. 112, 120505 (2014).

22. Roslund, J. et al. Wavelength-multiplexed quantum networks with ultrafast frequency combs. Nature Photon. 8, 109 (2014).

23. Xu, P. et al. Experimental studies of enhanced Raman scattering from a hexagonally poled $\mathrm{LiTaO}_{3}$ crystal. Phys. Rev. B 72, 064307 (2005).

24. van Loock, P. \& Furusawa, A. Detecting genuine multipartite continuous-variable entanglement. Phys. Rev. A 67, 052315 (2003).

25. Shen, Y. R. The Principles of Nonlinear Optics (Wiley, New York, 1984).

26. Kien, F. L. \& Hakuta, K. Parametric beating of a quantum probe field with a prepared Raman coherence in a far-off-resonance medium. Phys. Rev. A 67, 033808 (2003).

27. Ferraro, A. et al. Three-mode entanglement by interlinked nonlinear interactions in optical (2) media. J. Opt. Soc. Am. B 21, 1241-1249 (2004).

28. Guo, J., Zou, H. X., Zhai, Z. H., Zhang, J. X. \& Gao, J. R. Generation of continuous variable tripartite entanglement using cascaded nonlinearities. Phys. Rev. A 71, 034305 (2005).

29. Yu, Y. B. et al. Generation of three-mode continuous-variable entanglement by cascaded nonlinear interactions in a quasiperiodic superlattice. Phys. Rev. A 74 042332 (2006).

30. Walls, D. F. \& Milburn, G. J. Quantum Optics (Springer, Berlin, 1994).

31. Gardiner, C. W. Quantum Noise (Springer-Verlag, Berlin, 1991).

32. Pennarun, C., Bradley, A. S. \& Olsen, M. K. Tripartite entanglement and threshold properties of coupled intracavity down-conversion and sum-frequency generation. Phys. Rev. A 76, 063812 (2007).

33. Midgley, S. L. W., Bradley, A. S., Pfister, O. \& Olsen, M. K. Quadripartite continuous-variable entanglement via quadruply concurrent down-conversion. Phys. Rev. A 81, 063834 (2010).

34. Collett, M. J. \& Gardiner, C. W. Squeezing of intracavity and traveling-wave light fields produced in parametric amplification. Phys. Rev. A 30, 1386-1391 (1984)

35. Yu, Y. B., Xiao, M. \& Zhu, S. N. Three-colour entanglement generated by an injection-seeded nondegenerate optical parametric oscillator. Physics Letters A 375, 2278-2282 (2001).

36. Yu, Y. B. et al. Continuous-variable pair-entanglement frequency comb generated from an optical superlattice by enhanced Raman scattering. Phys. Rev. A 77, 032317 (2008).

37. Grosse, N. B., Bowen, W. P., McKenzie, K. \& Lam, P. K. Harmonic Entanglement with Second-Order Nonlinearity. Phys. Rev. Lett. 96, 063601 (2006).

38. Bremner, M. J., Mora, C. \& Winter, A. Are Random Pure States Useful for Quantum Computation? Phys. Rev. Lett. 102, 190502 (2009).

39. Gross, D., Flammia, S. T. \& Eisert, J. Most Quantum States Are Too Entangled To Be Useful As Computational Resources. Phys. Rev. Lett. 102, 190501 (2009).

40. Treps, N. et al. A quantum laser pointer. Science 301, 940 (2003).

41. Chalopin, B., Scazza, F., Fabre, C. \& Treps, N. Multimode nonclassical light generation through the optical-parametric-oscillator threshold. Phys. Rev. A 81, 061804(R) (2010). 
42. Huntington, E. H. et al. Demonstration of the spatial separation of the entangled quantum sidebands of an optical field. Phys. Rev. A 71, 041802 (2005).

\section{Acknowledgments}

This work is supported by National Natural Science Foundations of China (Nos. 10804059, 51275251, 11447211, and 11274187), Sate Key Program for Basic Research of China (No. 2012CB326407), Zhejiang Provincial Natural Science Foundation (No. Y6090488), Ningbo Natural Science Foundation (Nos. 2008A610006 and 2008A610004).

\section{Author contributions}

Y.B.Y. designed the study. X.M.C. directed the study. Y.B.Y. mainly executed the Project and wrote the manuscript. H.J.W., Z.T.S., J.W.Z., F.M.J., Z.Y. and Y.J.W. contributed to the discussion and edited the manuscript.

\section{Additional information}

Competing financial interests: The authors declare no competing financial interests.

How to cite this article: Yu, Y. et al. The generation of Continuous-Variable Entanglement Frequency Comb. Sci. Rep. 5, 7900; DOI:10.1038/srep07900 (2015).

(c) (1) $(-)$ This work is licensed under a Creative Commons Attribution-NonCommercialNoDerivs 4.0 International License. The images or other third party material in this article are included in the article's Creative Commons license, unless indicated otherwise in the credit line; if the material is not included under the Creative Commons license, users will need to obtain permission from the license holder in order to reproduce the material. To view a copy of this license, visit http:// creativecommons.org/licenses/by-nc-nd/4.0/ 